\title{
Ovulation induction with clomifene: a primary care perspective
}

\author{
Scott Wilkes, ${ }^{1}$ Alison Murdoch ${ }^{2}$
}

\begin{abstract}
${ }^{1}$ General Practitioner and Honorary Clinical Senior Lecturer, Institute of Health \& Society, Newcastle University, Newcastle upon Tyne, UK

${ }^{2}$ Consultant in Reproductive Medicine and Head of

Department, Newcastle Fertility Centre at LIFE, BioScience

Centre, International Centre for Life, Newcastle upon Tyne, UK
\end{abstract}

\section{Correspondence to}

Dr Scott Wilkes, Institute of Health \& Society, Newcastle University, Baddiley-Clark Building, Richardson Road, Newcastle upon Tyne NE2 $4 \mathrm{AX}, \mathrm{UK}$;

scott.wilkes@newcastle.ac.uk

Received 14 March 2011 Accepted 30 June 2011 Published Online First

7 August 2011

\begin{abstract}
Infertility affects one in seven couples during their lifetime. Approximately one-quarter of these will have an ovulatory disorder contributing to their inability to conceive. Ovulatory disorders represent the simplest form of infertility to treat, and where this is not a result of ovarian failure or poor ovarian reserve most women require ovulation induction with clomifene citrate (CC). This review aims to examine the role of CC in a general practice setting. CC is a simple, relatively safe, easily administered and welltolerated efficacious drug. There is, however, a $10 \%$ risk of multiple births associated with its use. CC has been used in general practice for many years and continues to be used. Currently, guidelines do not describe its use in the general practice setting and the evidence for monitoring its use with mid-luteal progesterone estimation or ultrasound scanning is conflicting.
\end{abstract}

\section{Introduction}

Clomifene citrate (CC) has been used widely for ovulation induction since the 1960 s on account of its effectiveness, ease of administration and minimal side effects. Ovulation disorders represent one-quarter of all couples who present to their general practitioner (GP) seeking assistance with reproduction. ${ }^{1-3}$ Approximately one-third of women seeking assistance for their fertility receive $\mathrm{CC}$ as a first-line treatment strategy. ${ }^{4}$ This article reviews the effectiveness, risks, clinical guidelines, monitoring strategies and the use of CC in general practice. It is beyond the scope of this review to consider other drugs including aromatase inhibitors and metformin.

\section{Literature search}

The Cochrane database, MEDLINE, ISI Web of Science and PubMed were searched for the period December 2000 to December 2010 with the following term 'clomifene' linked to 'infertility', 'ovulation induction', 'polycystic ovary

\section{Key message points}

Clomifene citrate (CC) is a simple, safe, effective and inexpensive drug used to induce ovulation. The risk of multiple pregnancy (mainly twin) is of the order of $10 \%$.

- Clinical guidelines support the use of CC in women with proven ovulatory dysfunction only. There is no role for CC in couples with a diagnosis of unexplained infertility.

$\checkmark$ One in ten couples who receive clomifene have their treatment initiated in general practice. CC use in general practice should be limited to six cycles only.

- CC cycles can be monitored using either mid-luteal progesterone or ultrasound scanning.

syndrome', 'primary care' or 'family practice'. A total of 2854 citations were found. Following removal of duplicate papers and scrutiny of the abstracts, 31 papers were relevant to the current review. In addition, the reference lists of the papers were reviewed and relevant articles, papers, guidelines and consensus statements were considered.

\section{Efficacy and effectiveness}

The efficacy of CC is well established through its capacity to produce increased levels of follicle-stimulating hormone (FSH). The mechanism of action of CC is thought to be via the blockage of estrogen receptors in the pituitary, interfering with negative feedback with resultant increase in $\mathrm{FSH}$, subsequent follicle maturation and ovulation. Its effectiveness in producing clinical pregnancies in differing cohorts of subfertile women has been the subject of study for many years.

Ovulation disorders are probably the easiest form of infertility to treat successfully ${ }^{5} 6$ with pregnancy rates approaching those of normally fertile couples. ${ }^{7}$ Ovulation induction with CC for women with oligomenorrhoea shows an increase in ovulation rate [odds ratio (OR) 7.47, 95\% confidence interval (CI) 3.24-17.23] and an increase in pregnancy rate per treatment cycle compared to placebo (OR $5.8,95 \%$ CI $1.6-21.5) .{ }^{8} \mathrm{CC}$ is simple to 
administer, cheap and effective, and there is evidence to suggest that it can be administered without inducing a withdrawal bleed in patients with oligomenorrhoea. ${ }^{9}$ It has also been shown to be similarly effective when administered in the luteal phase for women with polycystic ovary syndrome (PCOS). ${ }^{10}$

Women with ovulatory disorders represent approximately one-quarter of couples presenting with a fertility problem. ${ }^{1}$ Of those, $90 \%$ will be women with PCOS with the majority being overweight or obese. ${ }^{11} \mathrm{CC}$ is an effective first-line method of ovulation induction for both lean and obese women with PCOS. ${ }^{12}$ Over $75 \%$ of patients with PCOS will ovulate with $\mathrm{CC}^{13} 14$ with a conception rate of approximately $22 \%$ per cycle. ${ }^{15}$ The recommended first-line treatment for anovulatory women with PCOS is CC. ${ }^{16} 17$

There is no evidence from efficacy or effectiveness studies to support the use of CC in couples diagnosed with unexplained infertility. ${ }^{18} 19$ The World Health Organization (WHO) offers a treatment-orientated classification of ovulation disorders that it is useful to consider when addressing the efficacy of CC for this subgroup of patients. In summary, hypothalamic pituitary failure (WHO Group I), ovarian failure (WHO Group III) and hyperprolactinaemic ovulatory dysfunction (WHO Group IV) are unlikely to benefit from CC therapy because of its mechanism of action. The majority of patients with ovulatory dysfunction who will benefit from CC therapy will have hypothalamic-pituitary dysfunction (WHO Group II), predominantly PCOS.

\section{Risks}

CC is regarded as a relatively safe, easily administered, well-tolerated drug with few adverse effects. ${ }^{16}$ One of the theoretical risks of CC use is ovarian cancer. A recent Cochrane Review stated that excessive use of more than $12 \mathrm{CC}$ cycles may be associated with a three-fold increased risk in ovarian cancer. ${ }^{18}$ This was based upon a cohort study published in 1994 of 3837 infertile women where 11 women developed invasive or borderline malignant ovarian tumours compared to an expected incidence of $4.4 .^{20}$ Nine of these women were exposed to CC, giving a relative risk (RR) of 2.3 (95\% CI 0.5-11.4). Five of the nine women had prolonged exposure (i.e. more than 12 months), demonstrating a significant increase in the RR (RR 11.1, 95\% CI 1.5-82.3). A more recent case-control study has failed to demonstrate an association between borderline ovarian tumours and ovulation induction either with CC or with the more potent gonadotrophins. ${ }^{21}$ Similarly, a large Danish cohort study also failed to demonstrate an association between CC and ovarian cancer. ${ }^{22}$ To date there has been no conclusive evidence to support a causal association between ovarian cancer and CC use.

Multiple births are the most notable complications of assisted reproduction (ART) ${ }^{2324}$ and are more prevalent with gonadotrophin use. ${ }^{25}$ The consequences of multiple pregnancies are significant and include an increase in premature birth, low birth rate, Caesarean section, cerebral palsy, stillbirth, placental problems, gestational hypertension, pre-eclampsia and gestational diabetes. In the UK, $40 \%$ (25\% of pregnancies) of in vitro fertilisation (IVF) babies are twins ${ }^{26}$ with similarly high figures for ART in the USA. ${ }^{24}$ Multiple pregnancy risk with CC is approximately $10 \%,{ }^{27}$ the majority being twin pregnancies. This compares with a background incidence of spontaneous multiple pregnancies of approximately $1-2 \%,{ }^{2528}$ giving an estimated 10 -fold increased risk of multiple pregnancy with CC use. Of the multiple pregnancies induced by CC, $91 \%$ will be twin, $8 \%$ triplet and $1 \%$ quadruplet or higherorder pregnancies. ${ }^{25}$ Couples who are desperate for a child seem to accept this risk. When a cohort of 801 patients was asked to consider the risks and benefits of ART, $41 \%$ of patients were prepared to accept the risk of a multiple birth. ${ }^{29}$

\section{Policy and practice guidelines}

The Royal College of Obstetricians and Gynaecologists (RCOG) guideline 1999 stated that the prescribing of CC should not take place in general practice. ${ }^{30}$ Five years later the publication of the National Institute for Health and Clinical Excellence (NICE) guideline (currently adopted RCOG guideline) did not specifically support or refute general practice taking on the role of ovulation induction with CC but describes the patient groups in whom it should be used. ${ }^{31}$ NICE recommend CC as a first-line ovulation induction strategy for women with normogonadotrophic, hypothalamic-pituitary ovulatory dysfunction (predominately PCOS). Monitoring CC cycles with ultrasound was the view of the NICE Guideline Development Group:

NICE 1.6.1.4. Women undergoing treatment with clomifene citrate should be offered ultrasound monitoring during at least the first cycle of treatment to ensure that they receive a dose that minimises the risk of multiple pregnancy. ${ }^{31}$

The European Society of Human Reproduction and Embryology (ESHRE) recommend CC as firstline treatment for ovulation induction in anovulatory women with PCOS. ${ }^{16}$

$\mathrm{CC}$ is also recommended for use in obese, infertile women but account should be taken of the risks associated with a higher body mass index (BMI). ${ }^{32} \mathrm{~A}$ survey of fertility clinics in the UK showed that $51 \%$ of the units offered ovulation induction with CC for patients with a $\mathrm{BMI}<30 \mathrm{~kg} / \mathrm{m}^{2}, 47 \%$ for $\mathrm{BMI}<35 \mathrm{~kg} / \mathrm{m}^{2}$ and one unit offered treatment to women with a BMI $>40 \mathrm{~kg} / \mathrm{m}^{2} .^{33}$ The British Fertility Society recommends that fertility treatment should be deferred until the BMI $<35 \mathrm{~kg} / \mathrm{m}^{2}$, and treatment is preferable with a $\mathrm{BMI}<30 \mathrm{~kg} / \mathrm{m}^{2} .^{34}$ Women should aim for a normal BMI and the most 
effective form of treatment for obese, infertile women is weight reduction with a hypocaloric diet. ${ }^{32}$

If $\mathrm{CC}$ is used in primary care, the currently available literature suggests that treatment with CC should be limited to six cycles, should only be initiated following a full detailed assessment, and a maximum of 12 cycles in total should be administered following specialist evaluation. ${ }^{13} 15163135$

\section{Monitoring CC-induced cycles}

CC-stimulated cycles can be monitored with either serum mid-luteal progesterone estimation or ultrasound scanning in the follicular phase of the menstrual cycle. The latter is promoted as good clinical practice, which reduces the risk of multiple births and their associated complications. This is the view of the NICE Guideline Development Group ${ }^{31}$ but remains unproven.

Monitoring of CC cycles is commonly performed with serum mid-luteal progesterone in a district general hospital setting. ${ }^{36}$ In a general practice setting, monitoring of CC cycles with mid-luteal progesterone has been shown to be effective with a twin pregnancy rate of $4.6 \% .^{37}$

Some authors argue that monitoring of CC-induced cycles is not mandatory to ensure good patient outcomes. ${ }^{1638}$ There is currently no evidence to show that ultrasound monitoring prevents more multiple pregnancies than progesterone monitoring only.

GPs have access to both blood tests and transvaginal ultrasound through local commissioning arrangements and a recent Department of Health survey showed that the majority $(54 \%)$ of non-obstetric transvaginal ultrasound performed in the National Health Service (NHS) are requested by GPs. ${ }^{39}$ If the two monitoring methods are in a state of equipoise then it becomes important to address not only efficacy but also cost effectiveness. The risk to the patient is commissioning an inferior service and the risk to the NHS is the use of an expensive unnecessary service.

\section{Use in general practice}

Up to $28 \%$ of couples seeking assistance from their GP will ultimately receive CC as a first-line treatment strategy. ${ }^{14} \mathrm{CC}$ continues to be used in general practice in the UK and in primary care settings overseas. ${ }^{47} 4041$ A recent study in the UK showed that $9 \%$ of all couples with an ovulatory disorder who were offered CC as a first-line treatment had their treatment initiated by their GP. ${ }^{1}$ This is reflected in the fact that $65 \%$ of GPs feel that CC treatment should always be initiated by a hospital specialist. ${ }^{42}$ Some patients like the idea of their GP prescribing CC, stating that it is a relatively simple form of fertility treatment to administer as long as the prescribing GP has the appropriate knowledge and expertise. ${ }^{43} \mathrm{CC}$ use in general practice has been described in the literature by fertility specialists, ${ }^{5744}$ and management algorithms giving advice on prescribing and monitoring CC treatment have been published. ${ }^{75}$
Full investigation, diagnosis and management of female fertility in general practice have been described. ${ }^{45}$ It is beyond the scope of this review to comment on the treatment of women with identified endocrine causes of amenorrhoea or oligomenorrhoea. Ovulation induction with CC is indicated for women who have anovulation (excluding ovarian failure where women will present with amenorrhoea) and oligomenorrhoea, usually PCOS. There is no role for CC in couples with unexplained infertility. ${ }^{19}$

\section{Conclusions}

Simple problems with ovulation can be treated in general practice. ${ }^{7375}$ Whether the prescriber of CC is a GP or hospital specialist is not important. What matters is that CC is prescribed responsibly and monitored in accordance with current medical opinion and that patients are properly counselled about the risks.

Despite patient acceptance of the risk of multiple births, ${ }^{29}$ it is still important to reduce multiple births and the attendant risks and complications through patient education.

Perhaps of more importance for the GP acting as the patients' advocate is the prevention of patients (through non-referral) receiving gonadotrophin-stimulated cycles for clinical scenarios not supported in current guidance. Gonadotrophin stimulation treatment is recommended when ovulation cannot be induced with CC or as part of IVF treatment only. ${ }^{12}$

The use of CC does fall within the remit of general practice and has been used effectively in this setting. ${ }^{37}$ GPs may only see one to two infertile couples each year, ${ }^{1}$ which does not afford GPs the opportunity to become familiar with the skills necessary to manage infertile couples effectively. The challenge for primary care is to organise itself to deliver more effective management of the initial stages for the infertile couple which includes ovulation induction with CC. One strategy may include referrals from GPs to a GP who specialises in the management of subfertility. An opportunity exists to exploit this when general practices begin working together within commissioning consortia groups. ${ }^{46}$

If CC is prescribed in general practice its use should be limited to six cycles and the authors recommend monitoring with mid-luteal progesterone to allow for dose adjustment and tracking of ovulatory cycles associated with CC treatment. Further cycles should be at the discretion of the patient and fertility specialist following referral.

Research questions to arise from this review are:

- Do patient outcomes differ when CC is initiated in primary care, secondary care or tertiary care?

- Do patient outcomes differ when CC treatment is monitored with either mid-luteal progesterone or ultrasound scanning? 
Acknowledgements The authors are grateful to Professor Louise Robinson and Sally Black MBE for their comments on this manuscript.

\section{Competing interests None.}

Provenance and peer review Commissioned; externally reviewed.

\section{References}

1 Wilkes S, Chinn DJ, Murdoch A, et al. Epidemiology and management of infertility: a population-based study in UK primary care. Fam Pract 2009;26:269-274.

2 Hull MG, Glazener CM, Kelly NJ, et al. Population study of causes, treatment, and outcome of infertility. $\mathrm{Br} \mathrm{Med} J$ (Clin Res Ed) 1985;291:1693-1697.

3 Thonneau P, Marchand S, Tallec A, et al. Incidence and main causes of infertility in a resident population $(1,850,000)$ of three French regions (1988-1989). Hum Reprod 1991;6:811-816.

4 Marino JL, Moore VM, Rumbold AR, et al. Fertility treatments and the young women who use them: an Australian cohort study. Hum Reprod 2011;26:473-479.

5 Cahill DJ, Wardle PG. Management of infertility. BMJ 2002;325:28-32.

6 Hull MG, Savage PE, Bromham DR. Anovulatory and ovulatory infertility: results with simplified management. $\mathrm{Br} \mathrm{Med} J$ (Clin Res Ed) 1982;284:1681-1685.

7 Hargreave TB, Mills JA. Investigating and managing infertility in general practice. BMJ 1998;316:1438-1441.

8 Brown J, Farquhar C, Beck J, et al. Clomiphene and antioestrogens for ovulation induction in PCOS. [Update of Cochrane Database Syst Rev 2005;1:CD002249; PMID: 15674894]. Cochrane Database Syst Rev 2009;4:CD002249.

9 Farhi J, Orvieto R, Homburg R. Administration of clomiphene citrate in patients with polycystic ovary syndrome, without inducing withdrawal bleeding, achieves comparable treatment characteristics and outcome. Fertil Steril 2010;93:2077-2079.

10 Badawy A, Inany $\mathrm{H}$, Mosbah A, et al. Luteal phase clomiphene citrate for ovulation induction in women with polycystic ovary syndrome: a novel protocol. Fertil Steril 2009;91:838-841.

11 Balen AH, Dresner M, Scott EM, et al. Should obese women with polycystic ovary syndrome receive treatment for infertility? BMJ 2006;332:434-435.

12 Guzick DS. Ovulation induction management of PCOS. Clin Obstet Gynecol 2007;50:255-267.

13 Homburg R. Clomiphene citrate - end of an era? A minireview. Hum Reprod 2005;20:2043-2051.

14 Messinis IE. Ovulation induction: a mini review. Hum Reprod 2005;20:2688-2697.

15 Eijkemans MJ, Imani B, Mulders AG, et al. High singleton live birth rate following classical ovulation induction in normogonadotrophic anovulatory infertility (WHO 2). Hum Reprod 2003;18:2357-2362.

16 European Society of Human Reproduction and Embryology (ESHRE). Consensus on infertility treatment related to polycystic ovary syndrome. Thessaloniki. Hum Reprod 2008;23:452-477.

17 Zain MM, Jamaluddin R, Ibrahim A, et al. Comparison of clomiphene citrate, metformin, or the combination of both for first-line ovulation induction, achievement of pregnancy, and live birth in Asian women with polycystic ovary syndrome: a randomized controlled trial. Fertil Steril 2009;91:514-521.

18 Hughes E, Brown J, Collins JJ, et al. Clomiphene citrate for unexplained subfertility in women. [Update of Cochrane
Database Syst Rev 2000;3:CD000057; PMID: 10908459]. Cochrane Database Syst Rev 2010;1:CD000057.

19 Bhattacharya S, Harrild K, Mollison J, et al. Clomifene citrate or unstimulated intrauterine insemination compared with expectant management for unexplained infertility: pragmatic randomised controlled trial. BMJ 2008;337:a716.

20 Rossing MA, Daling JR, Weiss NS, et al. Ovarian tumors in a cohort of infertile women. $N$ Engl J Med 1994;331: 771-776.

21 Cusidó M, Fábregas R, Pere BS, et al. Ovulation induction treatment and risk of borderline ovarian tumors. Gynecol Endocrinol 2007;23:373-376.

22 Jensen A, Sharif H, Frederiksen K, et al. Use of fertility drugs and risk of ovarian cancer: Danish Population Based Cohort Study. BMJ 2009;338:b249.

23 Andersen AN, Goossens V, Ferraretti AP, et al. Assisted reproductive technology in Europe, 2004: results generated from European registers by ESHRE. Hum Reprod 2008;23: 756-771.

24 Wright VC, Chang J, Jeng G, et al. Assisted reproductive technology surveillance - United States, 2005. [Erratum appears in MMWR Surveill Summ 2009;58:203-4]. [Erratum appears in MMWR Surveill Summ 2008;57:1105]. MMWR Surveill Summ 2008;57:1-23.

25 Dickey RP. Strategies to reduce multiple pregnancies due to ovulation stimulation. Fertil Steril 2009;91:1-17.

26 Human Fertilisation and Embryology Authority. Multiple Births - Background and Statistics. 2011. http://www. hfea.gov.uk/docs/Multiple_births_background_and_statistics.pdf [accessed 21 January 2011].

27 Hamilton-Fairley D, Taylor A. Anovulation. BMJ 2003;327:546-549.

28 Ward Platt MP, Glinianaia SV, Rankin J, et al. The North of England Multiple Pregnancy Register: five-year results of data collection. Twin Res Hum Genet 2006;9:913-918.

29 Child TJ, Henderson AM, Tan SL. The desire for multiple pregnancy in male and female infertility patients. Hum Reprod 2004;19:558-561.

30 Royal College of Obstetricians and Gynaecologists. Royal College of Obstetricians and Gynaecologists Evidence-based Clinical Guidelines. Guideline Summary No. 2: The Initial Investigation and Management of the Infertile Couple. BJU Int 1999;83:636-645.

31 National Institute for Clinical Excellence (NICE). Fertility: Assessment and Treatment for People with Fertility Problems. London, UK: NICE, 2004.

32 Wilkes S, Murdoch A. Obesity and female fertility: a primary care perspective. J Fam Plann Reprod Health Care 2009;35:181-185.

33 Zachariah M, Fleming R, Acharya U. Management of obese women in assisted conception units: a UK survey. Hum Fertil (Camb) 2006;9:101-105.

34 Balen AH, Anderson RA. Impact of obesity on female reproductive health: British Fertility Society, Policy and Practice Guidelines. Hum Fertil (Camb) 2007;10:195-206.

35 British National Formulary (BNF). Clomifene Citrate. 2011. http://bnf.org/bnf/index.htm [accessed 22 January 2011].

36 Damodaran S, Mamatha L, Pheely M, et al. Experience of clomiphene use in a district general hospital. J Obstet Gynaecol 2010;30:46-48.

37 Stanford JB, Parnell TA, Boyle PC. Outcomes from treatment of infertility with natural procreative technology in an Irish general practice. [Erratum appears in J Am Board Fam Med 2008;21:583]. J Am Board Fam Med 2008;21:375-384. 
38 Legro RS, Barnhart HX, Schlaff WD, et al. Clomiphene, metformin, or both for infertility in the polycystic ovary syndrome. N Engl J Med 2007;356:551-566.

39 Department of Health. DH Knowledge \& Intelligence Team. Cancer Diagnostics Baselining Report. 2010. http:// www.dh.gov.uk/en/Publicationsandstatistics/Statistics/ Performancedataandstatistics/index.htm [accessed 6 May 2011].

40 Case AM. Infertility evaluation and management. Strategies for family physicians. Can Fam Physician 2003;49:1465-1472.

41 Whitman-Elia GF, Baxley EG. A primary care approach to the infertile couple. J Am Board Fam Pract 2001;14:33-45.

42 Souter VL, Penney G, Gorman DR. A survey of infertility practices in primary care in Scotland. Br J Gen Pract 1997;47:727-728.
43 Wilkes S, Hall N, Crosland A, et al. Patient experience of infertility management in primary care: an in-depth interview study. Fam Pract 2009;26:309-316.

44 Morrison J, Carroll L, Twaddle S, et al. Pragmatic randomised controlled trial to evaluate guidelines for the management of infertility across the primary care-secondary care interface. BMJ 2001;322:1282-1284.

45 Wilkes S, Fankam F. Review of the management of female infertility: a primary care perspective. Clinical Medicine Reviews in Women's Health 2010;2:11-23.

46 Department of Health. The White Paper. Equity and Excellence: Liberating the NHS. London, UK: Department of Health, 2010. 